\title{
Edge states in antiferromagnetic quantum spin chains
}

\author{
Tai-Kai Ng \\ Department of Physics, The Hong Kong University of Science and Technology, Clear Water Bay, Kowloon, Hong Kong
}

(Received 28 March 1994)

\begin{abstract}
The structure of edge states in general finite antiferromagnetic quantum spin chains with arbitrary spin value $S$ is discussed within the framework of the nonlinear-sigma model (NL $\sigma \mathrm{M}$ ) plus a topological $\theta$ term. Based on a large- $N$ theory of $\mathrm{SU}(N)$ quantum antiferromagnets and strong-coupling expansion, we argue that edge states with fractionalized spin quantum number $S^{\prime}$ exist in all spin chains with $S \geq 1$, with $S^{\prime}=S / 2$ for integer spin chains and $S^{\prime}=(S-1 / 2) / 2$ for half-integer spin chains.
\end{abstract}

Recently, there has been much interest in nontrivial edge states in quantum-mechanical systems, most noticeably in the study of the fractional quantum Hall effect (FQHE). ${ }^{1-3}$ Another system of interest is the $S=1$ antiferromagnetic spin chain, where edge states with fractionalized spin quantum number $S^{\prime}=\frac{1}{2}$ are being observed experimentally. ${ }^{4,5}$ Since the low-energy physics of both systems is believed to be described by field theories with topological terms, it is natural to ask whether the edge states in these systems are intrinsically related to their topological characters. In this paper we shall address this problem for quantum spin chains from two directions, large- $N$ theory of $\mathrm{SU}(N)$ quantum antiferromagnets and strong-coupling expansion. We shall first consider integer-S spin chains and show that the $S^{\prime}=S / 2$ edge state is a direct consequence of the existence of topological terms in the effective Hamiltonian in the large$N$ theory. As an indirect confirmation of the theory, the phenomenon of weak-dimerization will be discussed. We shall then extend our discussion to spin chains with halfinteger spin value $S$, where based on a strong-coupling expansion, we argue that edge states with fractionalized spin-quantum numbers $S^{\prime}$ exist for all $S \geq 1$, with $S^{\prime}=S / 2$ for integer spin chains and $S^{\prime}=(S-1 / 2) / 2$ for half-integer spin chains. The structure of edge states in spin chains with arbitrary value of topological angle $\theta$ will also be discussed.

The relation of edge states in integer- $S$ spin chains to topological term in the low-energy continuum theory $[O(3)$ nonlinear sigma model] can be most easily visualized by looking at the Berry phase contribution to the continuum theory. ${ }^{6,7}$ For a finite spin chain of length $L$, the Berry phase contribution to the effective action is ${ }^{6,7}$

$$
\begin{aligned}
S_{\mathrm{BP}} & =\hbar S \sum_{i}(-1)^{i} \Omega\left(x_{i}\right) \sim \frac{\hbar S}{2} \int \frac{\partial \Omega(x)}{\partial x} d x \\
& =\frac{\hbar S}{2}\{[\Omega(L)-\Omega(0)]+4 \pi Q\},
\end{aligned}
$$

where $\Omega(x)$ is the solid angle subtended by the close path on the surface of a unit sphere defined by the time evolution of a unit vector $n$ at $x$. $Q$ is an integer measuring the number of times the spin configuration $\mathbf{n}(x, t)(x=0$ to $L$, $t=-\infty$ to $\infty)$ covers the surface of the unit sphere. ${ }^{6,7}$ Notice that for integer spin chains, $S \times Q$ is always an in- teger and $\exp (i 2 \pi S Q)$ is always one, i.e., the term $\hbar 2 \pi S Q$ has no effect on the path integral and can always be eliminated. With periodic boundary condition the edge contributions at $x=0$ and $x=L$ cancel, and the Berry phase action has no effect. For open chains, contributions at end points survive with $S_{\text {end points }}=\hbar(S / 2)[\Omega(L)-\Omega(0)]$, which can be interpreted as the Berry phase of two free spins with spin magnitude $S / 2$, located at the end points $x=0$ and $L$, respectively. ${ }^{8}$ Performing a strong-coupling expansion to lowest order, ${ }^{9}$ we find that the system is everywhere a spin singlet except at the end points where the Berry phases give rise to two free spins with spin magnitude $S / 2$ located at each end of the spin chain. ${ }^{8,9}$

The edge states can also be understood in a Gaussian theory in the $\mathrm{CP}^{1}$ representation of the $\mathrm{O}(3) \sigma$ model where the low-energy effective Lagrangian is written as $^{7,10}$

$$
L=\frac{1}{g}\left\{\left|\left(\partial_{\mu}+i A_{\mu}\right) Z\right|^{2}-i \frac{\theta}{2 \pi} \epsilon^{\mu \nu} \partial_{\mu} A_{v}\right\},
$$

where $Z$ is a two-component complex spinor field $\left[\mathbf{n}=\left(Z^{*} \sigma Z\right)\right.$ with $\left.|z|^{2}=1\right], g \sim 2 / S$, and $\theta=2 \pi S$. The first term is the usual $O(3) \sigma$ model whereas the second term is the topological term discussed above. The advantage of the formulation is that within the Gaussian approximation, we can study edge states for spin chains with an arbitrary value of $\theta$. The Gaussian theory is usually formulated in a large- $N$ expansion ${ }^{7,10,11}$ of the $\mathbf{C P}^{N-1}$ model where the two-component spinor field is replaced by an $N$-component $z$ field. A large- $N$ expansion can be performed on this model with the help of a Lagrange multiplier field $\lambda$ to enforce the constraint. To lowest order (Gaussian approximation), we find $N$ branches of free massive $z$ bosons with dispersion $\omega_{k}^{2}=m^{2}+(c k)^{2}$ where $m \sim \exp (-\pi / N g)$ is the mass gap, $k$ the momentum vector of the bosons, and $c$ is the spin-wave velocity.

To order $1 / N$, an additional effective Lagrangian $\delta L$ is generated for the gauge field ${ }^{7,10,11}$

$$
\delta L=\frac{1}{4 e^{2}} F_{\mu v}^{2}
$$

where $e^{2} \sim m^{2} / N$ and the $z$ bosons are coupled to the gauge field through Lagrangian (2). Notice that $U(1)$ gauge theory has linear Coulomb force in one dimension 
and is confining. Thus we expect pairing of the $z$ bosons in the $\mathrm{CP}^{N-1}$ model. For $N=2$ and in the original spin language, we expect that $S=\frac{1}{2}$ elementary excitations in the large- $N$ theory are confined in pairs, implying that the "real" elementary excitations have a spin magnitude of one.

The effect of $\theta$ term has been discussed by Coleman ${ }^{10}$ in the infinite chain case where edge states do not exist. We shall generalize his discussion to finite chains in the following. Notice that $i \varepsilon^{\mu v} \partial_{\mu} A_{v}$ is just the electric field of the $\mathrm{U}(1)$ gauge theory and the $\theta$ term, linear in the electric field, can be interpreted as corresponding to a uniform external electric field with magnitude $E_{\text {ext }}=\theta /(2 \pi) e$, imposed on the quantum spin chain. For a chain with open ends, the electric field can be interpreted as coming from external charges, $\pm(\theta / 2 \pi) e$, placing at opposite ends of the one-dimensional universe, which produces the electric field. ${ }^{7,10}$ For integer $S=n, \theta=2 n \pi$ and the external charge is ne. Now we may ask what is the ground state of this system. One possible solution is that the electric field remains constant throughout the chain, and the energy of such a solution $\sim E_{\text {ext }}{ }^{2} L \sim(\mathrm{nm})^{2} L / N$, where $L$ is the length of the chain. Alternatively, a solution with much lower energy can be constructed by nucleating $n z$ boson charge pairs from the vacuum going off to the ends of the spin chain to screen the external field. The energy cost of such a solution is of order $(2 n) m$, which is much lower than the energy of first solution as $L$ becomes large. For $N=2$, each $z$ boson carries spin $\frac{1}{2}$, and the $n$ bosons nucleated at each end together forms edge state with spin quantum number $S^{\prime}=S / 2,{ }^{8}$ in exact agreement with general expectation.

It is clear in the above picture that the presence of an effective one-dimensional gauge theory is crucial for the explanation of the edge states. It is thus natural to ask whether there exists other physically observable effects associated with the gauge field which one can test? Read and Sachdev ${ }^{12}$ have shown in a $1 / N$ expansion that in quantum spin chains, the effective electric field is directly proportional to the dimerization (spin-Peierls) effect $\left(\mathbf{E} \sim \mathbf{q}=\left\langle\mathbf{S}_{i} \cdot \mathbf{S}_{i+1} \cdot \mathbf{S}_{i} \cdot \mathbf{S}_{i-1}\right\rangle\right)$ and a spin chain carrying a uniform electric field is equivalent physically to a uniformly dimerized spin chain. The equivalence between electric field and dimerization has an interesting consequence on the behavior around the edges of spin chains. Notice that the $z$ bosons in the large- $N$ expansion have a gap $m$ in the excitation spectrum, implying that there exists a characteristic "size" of the boson wave function, $\xi \sim m^{-1}$, and the edge states in the quantum spin chains are "smeared out" around the edges within a distance $\sim \xi$. The external electric field thus penetrates into the spin chain and is screened out completely only at a distance $x>\xi$ away from ends of spin chain. That immediately implies that regions around ends of spin chains are dimerized with magnitude of dimerization decaying exponentially with characteristic length $\sim \xi$ away from ends of spin chain. ${ }^{8}$ Notice that this "weakdimerization" effect should not be observed in phases of quantum spin chains which are not described as a $U(1)$ gauge theory. Indeed, exact diagonalization studies ${ }^{9}$ on $S=1$ finite quantum spin chains have confirmed such a picture where it is found that weak-dimerization effects exist only in the Haldane phase of quantum spin chains with the Hamiltonian

$$
H=\sum_{i} \mathbf{S}_{i} \cdot \mathbf{S}_{i+1}+D\left(S_{i z}\right)^{2},
$$

and is absent in the large- $D$ phase where edge states do not exist.

Now let us extend our discussion to arbitrary value of $\theta$ in the topological term within the large- $N$ approximation. Following Coleman, ${ }^{7,10}$ for general $\theta \neq 2 n \pi$, the "external charge" is not quantized and the background electric field cannot be screened completely by nucleating integer charge pairs. Finite electric field always remains in the spin chain meaning that the spin chain is dimerized for general $\theta \neq 2 n \pi$. Increasing $\theta$ from $\theta=0$, nucleation of charge pairs becomes energetically favorable as $\theta \geq \pi$, in which case one boson pair is nucleated. The external charge is overscreened resulting in an effective external charge $e^{\prime}=[\theta /(2 \pi)-1] e$ and a background electric field $\mathbf{E} \sim \mathbf{e}^{\prime}$ which is reversed in direction in space. Physically, an edge state with spin quantum number $S^{\prime}=\frac{1}{2}$ is formed at $\theta=\pi$ and the dimerization order parameter $\mathbf{q}=\left\langle\mathbf{S}_{i} \cdot \mathbf{S}_{i+1}-\mathbf{S}_{i} \cdot \mathbf{S}_{i-1}\right\rangle$ reversed in sign abruptly when $\theta$ increases through $\pi$, i.e., a first-order transition occurs at $\theta=\pi$. The magnitude of dimerization goes to zero continuously as $\theta$ approaches $2 \pi(\mathbf{E} \rightarrow 0)$ and increases in magnitude (but with direction reversed) again when $\theta$ increases further until $\theta=3 \pi / 2$, in which case another pair of bosons is nucleated and the spin quantum number of the edge states jumps from $\frac{1}{2}$ to 1 as $\theta$ increases across $3 \pi / 2$, with the dimerization order parameter $\mathbf{q}$ reversed in sign abruptly again. Repeating the above construction for general values of $\theta$, we obtain the following picture: for general $\theta \neq 2 n \pi$, spin chains are dimerized. The dimerization parameter $\mathrm{q}$ jumps from $+e / 2$ to $-e / 2$ every time $\theta$ crosses $(2 n+1) \pi$, and is increasing continuously from $-e / 2$ to $+e / 2$ in between. At each transition around $\theta=(2 n+1) \pi$, the edge state's spin quantum number increases by $\frac{1}{2}$. This result is summarized in Fig. (1). Notice that in this picture, the edge states play a vital role in recovering the periodicity of $\mathbf{q}$ in $\theta$ $[\mathbf{q}(\theta)=\mathbf{q}(\theta+2 \pi)]$ in the bulk spin chain. The periodicity of ground-state properties in $\theta$ is required in spin chains with periodic boundary conditions. Without the edge states, the external electric field will not be screened and $\mathrm{q}$ will increase monotonically with increasing $\theta$ [see Fig. (1)]. The (bulk) ground-state properties will not be periodic in $\theta$. Thus edge states are "required" in spin systems with topological terms to ensure that the (bulk) properties of open spin chains are the same as properties of corresponding spin chains with periodic boundary conditions.

It is interesting to point out that the above edge-state picture can also be obtained by following an analysis very similar to the one for edge states in FQHE. ${ }^{1-3}$ Notice that the effective Lagrangian $(2)+(3)$ is in fact applicable only for infinite chains and one may ask as in FQHE how the effective Lagrangian should be modified if the system is finite, assuming that the finite system is still described by an effective $U(1)$ scalar field theory. As discussed above, the $\theta$ term introduces a background electric field 


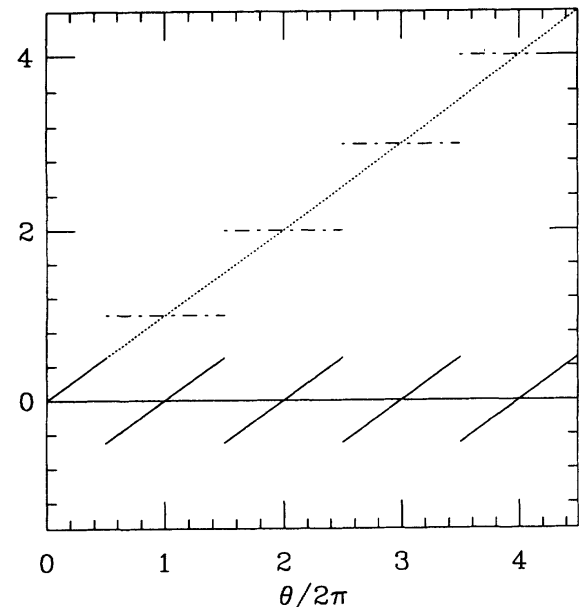

FIG. 1. Dimerization parameter $q$ and number of edge-state bosons $m_{\text {edge }}$ in large- $N$ theory of quantum spin chains as a function of topological angle $\theta$. $q$ is represented by a solid line, and $m_{\text {edge }}$ represented by a dot-dashed line. The spin magnitude of edge state is $S^{\prime}=m_{\text {edge }} / 2$. Notice the jumps in $m_{\text {edge }}$ and $q$ at $\theta=(2 n+1) \pi$ points. The dotted line represents the dimerization parameter $q$ if edge states are absent. Notice that the periodicity of $q$ in $\theta$ is recovered by creation of edge states.

in the system. For a finite system, a boundary action has to be introduced to confine the background electric field in the system. It is easy to see that the external charge in Coleman's picture is just what is required to confine the electric field and the edge states follow subsequently from our analysis.

The above picture is exact in the large- $N$ limit of $\mathrm{SU}(N)$ quantum antiferromagnets. For the physical case $N=2$, it is believed that the large- $N$ results are qualitatively correct for most values of $\theta$, except at regions around $\theta=(2 n+1) \pi$ (corresponding to half-integer spin chains), ${ }^{7}$ where it is believed that the transition across the $\theta=(2 n+1) \pi$ point should be a second-order transition. Moreover, from exact Bethe ansatz results for $S=\frac{1}{2}$ spin chains, we know that the (bulk) excitation spectrum should be gapless at the point $\theta=\pi$, whereas it is predicted to have a gap $\sim m$ in the large- $N$ theory. ${ }^{7}$ Notice, however, that although predictions on the bulk properties of half-integer spin chains are wrong, the predicted edgestate structure from large- $N$ theory may still be valid, since it appears that edge states are required for a very general reason in the large- $N$ theory.

To study edge states in half-integer spin chains, we consider the Berry phase contribution to the effective action again. Equation (1) for $S_{\mathrm{BP}}$ is still valid, except that $S$ is now a half-integer and $\exp (i 2 \pi S Q)$ is equal to $(-1)^{Q}=\exp (i \pi Q)$. Thus Eq. (1) can be rewritten for half-integer spin chain as

$$
\begin{aligned}
S_{\mathrm{BP}}=\hbar & \left\{\frac{S}{2}[\Omega(L)-\Omega(0)]+\pi Q\right\}, \\
=\hbar & \left\{\frac{1}{2}\left\{S-\frac{1}{2}\right][\Omega(L)-\Omega(0)]\right. \\
+ & \left.\frac{s}{2}[\Omega(L)-\Omega(0)+4 \pi Q]\right\},
\end{aligned}
$$

where $s=\frac{1}{2}$. Notice that the second term in Eq. (3a) is just the Berry phase action of a finite $S=\frac{1}{2}$ spin chain in the continuum limit. Thus Eq. (3a) can be considered as the continuum limit of a discrete antiferromagnetic spin model with Berry phase action

$$
S_{\mathrm{BP}}=\hbar \sum_{i}(-1)^{i} S_{i} \Omega\left(x_{i}\right) \text {, }
$$

where $S_{i}=\frac{1}{2}+(S-1 / 2) / 2$ on sites $x=0$ and $L$, and $S_{i}=\frac{1}{2}$ for sites in between. Now performing a strongcoupling expansion as before, we obtain a (bulk) $s=\frac{1}{2}$ spin chain ${ }^{13}$ coupling to "impurity" spins of magnitude $S_{\text {im }}=(S-1 / 2) / 2+1 / 2$ at the end points $x=0$ and $L$. This result can also be obtained simply by noting that Eq. (3) plus $N L \sigma M$ is just the continuum model of a $s=\frac{1}{2}$ spin chain coupled to two impurity spins $S_{\text {im }}$ at the end points as described above. Models of an $s=\frac{1}{2}$ spin chain coupled to impurity spins have been investigated in detail by Eggert and Affleck ${ }^{14}$ where they showed that a Kondo-type effect occurs in the antiferromagnetic coupling case. For $S_{\text {im }}>\frac{1}{2}$, the Kondo effect cannot screen the impurity spin completely and a "free" spin of magnitude $S_{\mathrm{im}}-\frac{1}{2}$ is left. Following their analysis, we conjecture that the impurity spin $S_{\text {im }}$ cannot be screened completely by the $s=\frac{1}{2}$ spin chain as long as $S_{\mathrm{im}}>\frac{1}{2}$, and edge states with spin magnitude $S_{\mathrm{im}}-\frac{1}{2}$ remain at edges of the spin chain. Correspondingly, in the original finite spin chain model, we conjecture that edge states exist in all finite spin chains with spin magnitude $S>\frac{1}{2}$, with magnitude $S^{\prime}=S / 2$ for integer spin chains, and $S^{\prime}=S_{\text {im }}-\frac{1}{2}=(S-1 / 2) / 2$ for half-integer spin chains. Notice that this result is in agreement with the predicted edge-state structure obtained from large- $N$ theory. Physically, the existence of edge states in finite half-integer spin chains can be understood by assuming that the ground-state wave function of a half-integer spin chain is just a superposition of wave function of an $s=\frac{1}{2}$ spin chain on a spin $S-\frac{1}{2}$ valence bond solid. ${ }^{15}$ The observed edge states in the half-integer spin chains are originating from edge states of the corresponding valence bond solids.

Now let us go back to the NL $\sigma \mathrm{M}$ [Eq. (2)] and study the behavior of edge states around the transition regions $\theta \sim(2 n+1) \pi$. Physically, NL $\sigma$ M's with $\theta$ values slightly deviating from $(2 n+1) \pi$ can be considered as continuum models of $S=n+\frac{1}{2}$ spin chains with alternating interaction $J_{i, i+1}=J\left[1+\gamma(-1)^{i}\right]$, $^{7}$ when $\gamma$ is small. In this limit, $\theta=2 \pi S(1+\gamma)$ (Ref. 7) and moving across $\theta=(2 n+1) \pi$ point correspond to half-integer spin chains with alternating $J_{i, i+1}$ when $\gamma$ changes sign. For an infinite chain with nonzero $\gamma$, the spin chain is dimerized and a spin gap $\Delta_{g}$ develops in the spin-excitation spectrum ${ }^{7}$ with $\Delta_{g} \sim|\gamma|^{3 / 2}$. The sudden appearance of additional edge states in the finite chain when $\gamma$ changes sign can be understood in the dimerization picture by considering a $S=\frac{1}{2}$ finite chain with $2 m$ sites. The spin chain is dimerized completely and no edge states appear if $J_{i, i+1}$ is finite for $i=2 k+1$ and is zero for $i=2 k$, where $k=$ integer. However, two free $S=\frac{1}{2}$ spins will be left at 
the edges if $J_{i, i+1}$ is finite for $i=2 k$ and is zero for $i=2 k+1$ since in this case, the first and last sites are decoupled from the rest of the spin chain. For $\gamma$ small, the edge spins are not completely decoupled from the spin chain and edge states which decay into the spin chain with a finite coherence length $\xi \sim \Delta_{g}^{-1} \sim|\gamma|^{-3 / 2}$ will be formed. Notice that $\xi \rightarrow \infty$ as $\gamma \rightarrow 0$, indicating that the points $\theta=(2 n+1) \pi(\gamma=0)$ are second-order critical points at which new $s=\frac{1}{2}$ edge states are formed, in agreement with expectations from our previous analysis. Physically, spin chains with alternating $J$ s can be formed in systems with Peierls instability. ${ }^{16}$ Thus the edge-state behavior predicted here can be tested experimentally. For example, for a collection of randomly broken $S=\frac{1}{2}$ spin chains, Curie-Weiss-type behavior in spin susceptibility is expected to be found at the lowtemperature dimerized phase, and is expected to be absent at the high-temperature normal phase. More generally, we predict that for (undimerized) antiferromagnetic quantum spin chains with spin magnitude $S>\frac{1}{2}$, Curie-Weiss behavior in spin-susceptibility will be observed upon doping by nommagnetic impurities ${ }^{5}$ because of generation of edge states.

It is important to point out that the edge states studied in this paper are intrinsic "edge" effects, but not finitesize effects associated with finite spin chains. One way to see this is to observe that both the "external charges" in the large- $N$ theory and the extra Berry phase appearing at the ends of a spin chain will disappear once a periodic boundary condition is imposed, no matter what the length of the spin chain. More generally, one may generalize our analysis to study edge states in semi-infinite spin chains where finite-size effects are automatically excluded. It is easy to see that our predicted edge states picture remains in semi-infinite chains, indicating that the edge states discussed in this paper are intrinsic edge effects.

Summarizing, in this paper we propose a general theory for edge states in finite antiferromagnetic quantum spin chains within the framework of the NL $\sigma$ M. The model is believed to describe the low-energy dynamics of infinite quantum spin chains correctly. For finite systems, the validity of this approximation is not $a$ priori clear. For finite integer spin chains, the model reproduces known results for edge states. Moreover, numerical solutions of the Schwinger-boson-mean-field theory ${ }^{17}$ and exact diagonalization study ${ }^{9}$ confirm that the NL $\sigma \mathrm{M}$ is the correct starting point ${ }^{8}$ for describing edge states (confirmation of weak dimerization). Within the NL $\sigma \mathrm{M}$, we find that the presence of edge states is a direct consequence of the topological character of spin chains and is not restricted to integer spin chains as is believed widely. Results obtained in this paper have direct experimental consequences and can be tested numerically especially for the more interesting case of half-integer spin chains.

The author thanks A. Tanaka for many interesting discussions which led to this work and for the hospitality of the Aspen Center for Physics for which part of this work is being done.
${ }^{1}$ X. G. Wen, Phys. Rev. Lett. 64, 2206 (1990).

${ }^{2}$ A. H. MacDonald, Phys. Rev. Lett. 64, 220 (1990).

${ }^{3}$ X. G. Wen, Phys. Rev. B 43, 11025 (1991).

${ }^{4}$ M. Hagiwara, K. Katsumata, I. Affleck, B. I. Halperin, and J. P. Renard, Phys. Rev. Lett. 65, 3181 (1990).

${ }^{5}$ S. H. Glarum, S. Geschwind, K. M. Lee, M. L. Kaplan, and J. Michael, Phys. Rev. Lett. 67, 1614 (1991).

${ }^{6}$ F. D. M. Haldane, Phys. Lett. 93A, 464 (1983); I. Affleck, Nucl. Phys. B257, 397 (1985).

${ }^{7}$ See I. Affleck, in Field Theory Methods and Quantum Critical Phenomena, edited by E. Brezin and J. Zinn-Justin (NorthHolland, Amsterdam, 1990).
${ }^{8}$ T. K. Ng, Phys. Rev. B 47, 11575 (1993).

${ }^{9}$ A. Tanaka, M. Oshikawa, and Y. Hatsugai (unpublished).

10S. Coleman, Ann. Phys. (N.Y.) 101, 239 (1976).

${ }^{11}$ E. Witten, Nucl. Phys. B149, 285 (1979).

${ }^{12}$ N. Read and S. Sachdev, Phys. Rev. Lett. 62, 1694 (1989).

${ }^{13}$ N. Read and R. Shankar, Nucl. Phys. B336, 457 (1990).

${ }^{14}$ S. Eggert and I. Affleck, Phys. Rev. B 46, 10866 (1992).

${ }^{15}$ I. Affleck, T. Kennedy, E. H. Lieb, and H. Tasaki, Phys. Rev. Lett. 59, 799 (1987).

${ }^{16}$ D. Guo, T. Kennedy, and S. Mazumdar, Phys. Rev. B 41, 9592 (1990).

${ }^{17}$ T. K. Ng, Phys. Rev. B 45, 8181 (1992). 